\title{
Tiling Groups: New Applications in the Triangular Lattice
}

\author{
E. Rémila \\ Institut Universitaire de Technologie de Roanne, \\ 20 Avenue de Paris, 42334 Roanne, France \\ remila@univ-st-etienne.fr
}

Communicated by Bruce L. Rothschild

\begin{abstract}
We first give a new presentation of an algorithm, from W. Thurston, to tile polygons with "calissons" (i.e., lozenges formed from two cells of the triangular lattice $\Lambda$ ). Afterward, we use a similar method to get a linear algorithm to tile polygons with $m$-leaning bars (parallelograms of length $m$ formed from $2 m$ cells of $\Lambda$ ) and equilateral triangles (whose sides have length $m$ ) and we produce a quadratic algorithm to tile polygons with $m$-leaning bars.
\end{abstract}

\section{Introduction}

Given a finite set of basic tiles (which are called prototiles), how can a "good" algorithm of tiling (i.e., an algorithm in polynomial time which, given a figure of the plane, gives a tiling of this figure with copies of the prototiles or indicates that the figure cannot be tiled) be exhibited? Conway and Lagarias [CL] defined tiling groups as a tool to study tiling problems. Their method gives a lot of necessary conditions for a simply connected figure to be tileable (see [LR] and [P]). Their work has been prolonged by Thurston [T] who introduced (in some particular cases) the notion of height function associated with a tiling. Using this new notion, algorithms to tile a simply connected figure with dominoes, or with "calissons," have been produced. Thurston's ideas have again been taken by $\mathrm{C}$. and $\mathrm{R}$. Kenyon [KK], which obtained an algorithm to tile a simply connected figure with $m$-bars (rectangles of length $m$ and unit width).

In Section 2 we recall the notions introduced by J. H. Conway, J. C. Lagarias, and W. P. Thurston. We point out the notions of "overtilings" and "tiling projections" which make the method of tiling more natural. In Section 3 these notions are illustrated by an example of W. P. Thurston: the tiling with calissons. We give an algebraic presentation when W. P. Thurston used geometrical arguments. 
Our purely algebraic framework permits us to extend the notion of a height function to other examples: in Section 4 we produce a linear algorithm of tiling of a simply connected figure with "leaning bars" of length $m$ (i.e., parallelograms with large sides of length $m$ and smell sides of unit length) and equilateral triangles with sides of length $m$. In Section 5 we produce a quadratic algorithm of tiling with leaning bars, simultaneously using four height functions. These new algorithms are the main results of this paper. They generalize and completely prove the algorithms announced in a previous paper [R].

\section{Tiling Groups and Tiling Graphs}

\subsection{Tilings}

Let $\Lambda$ be the triangular lattice of the Euclidean plane. A (finite) figure $F$ of $\Lambda$ is a (finite) union of (closed) cells of $\Lambda$. A figure $F$ is simply connected if $F$ and its complement $\mathbb{R}^{2}-F$ are both connected. A finite simply connected figure $F$ is called a polygon of $\Lambda$. The boundary of a polygon $P$ canonically induces a cycle in $\Lambda$ called the boundary cycle of $P$.

A set $S$ of prototiles is a fixed finite set of polygons of $\Lambda$. A tile is a translated copy of a prototile. A tiling $T$ of a figure $F$ is a set of tiles included in $F$, with pairwise disjoint interiors, such that the union of the tiles of $T$ equals $F$.

\subsection{Cayley Graphs}

Let $\Sigma$ be the set $\left\{a, b, c, a^{-1}, b^{-1}, c^{-1}\right\}$, let $F_{a, b, c}$ be the free group generated by $A=$ $\{a, b, c\}$ and let $s$ denote the canonical surjection from the language $\Sigma^{*}$ to $F_{a, b, c}$.

Let $R$ be a finite set of words of $\Sigma^{*}$. Group $N_{R}$ denotes the normal group of $F_{a, b, c}$ generated by the elements of $s(R)$ and $G_{R}$ denotes the quotient group $F_{a, b, c} / N_{R}$. The Cayley graph $C_{R}$ is the directed graph with labeled edges such that:

(i) the vertices of $C_{R}$ are the elements of $G_{R}$;

(ii) the set of labels is $\{a, b, c\}$; and

(iii) for each pair $\left(g, g^{\prime}\right)$ of elements of $\left(G_{R}\right)^{2}$ and each element $u$ of $A$, there exists an edge of $C_{R}$ from $g$ to $g^{\prime}$, labeled by $u$, if and only if $g u=g^{\prime}$.

The underlying graph of $\Lambda$ can be seen as the Cayley graph $C_{\text {triangle }}=C_{R}$, with $R=\{a b c, a c b\}$ (see Fig. 1), each element of $A$ being associated to a unit step. Hence, each vertex of $\Lambda$ is identified to an element of $G_{R}$ and has three incoming edges, one of each label, and three outgoing edges, one of each label.

Let $p=\left(v_{0}, v_{1}, \ldots, v_{p}\right)$ be a path of $\Lambda$, i.e., a sequence of vertices such that, for each integer $i$, with $0 \leq i<p$, there exists an element of $\Sigma$ such that $v_{i} u_{i}=v_{i+1}$. The path word $w(p)$ is the word $u_{0} u_{1}, \ldots, u_{p-1}$. Moreover, if $p$ is a boundary cycle of a polygon $P$, we say that $w(p)$ is a contour word of $P$. Let $S=\left\{t_{1}, t_{2}, \ldots, t_{p}\right\}$ be a set of prototiles. The tiling group of $S$ is the group $G_{\text {tile }}=G_{R_{S}}$, where $R_{S}=\left\{w_{1}, w_{2}, \ldots, w_{p}\right\}$ and for each integer $i$ such that $1 \leq i \leq p, w_{i}$ is a contour word of $t_{i}$. The tiling Cayley graph of $S$ is the graph $C_{\text {tile }}=C_{R_{S}}$. 


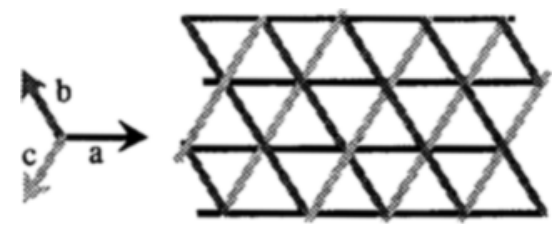

Fig. 1. Cayley graph $C_{\text {triangle }}$.

\subsection{Overtilings and Tiling Projections}

Let $T$ be a tiling of a figure $F$. The graph $G_{T}=\left(V_{T}, E_{T}\right)$ of $T$ is the subgraph of $C_{\text {triangle }}$ such that $E_{T}$ is the set of the edges which are included on the boundary of a tile of $T$, and $V_{T}$ is the set of the extremities of the edges of $E_{T}$.

Definition 2.1. Let $S$ be a set of prototiles, $F$ a figure of $\Lambda, T$ a tiling of $F$ generated by the prototiles of $S$, and let $G_{\text {tile }}$ denote the tiling group associated to the set $S$ of prototiles. An overtiling constructed over $T$ is a mapping $f_{T}$ from the set $V_{T}$ of the vertices of $G_{T}$ to $G_{\text {tile }}$ such that, for each pair $(v, u)$ of $V_{T} \times E_{T}$, the equality $f_{T}(v u)=f_{T}(v) u$ holds.

Proposition 2.2 (J. H. Conway). Let $F$ be a figure of $\Lambda, T$ a tiling of $F, v_{0}$ a vertex of $G_{T}$, and $v_{0}^{\prime}$ a vertex of $G_{\text {tile }}$.

If $F$ is connected (resp., simply connected), then there exists at most one (resp., exactly one) overtiling $f_{T}$ constructed over $T$ such that $f_{T}\left(v_{0}\right)=v_{0}^{\prime}$.

Sketch of the Proof. Function $f_{T}$ can easily be constructed successively exploring the contour of each tile: function $f_{T}$ is first defined on the vertices of the boundary of a tile $t_{0}$, which contains $v_{0}$. Afterwards, $f_{T}$ is defined on the vertices of a tile $t_{1}$ which has a common vertex with $t_{0}$ and so on (i.e., $f_{T}$ is defined on a tile $t_{i}$ which has a common vertex with the figure tiled by $\left.\left\{t_{0}, t_{1}, \ldots, t_{i-1}\right\}\right)$. This method proves that there exists at most one overtiling $f_{T}$ for $F$ being connected.

If $F$ has some holes, a conflict (i.e., a vertex $v$ such that two distinct values of $f_{T}(v)$ are obtained) can arise, which yields that there is no overtiling. Such a conflict cannot arise when $F$ is simply connected, since the sequence $\left(t_{0}, t_{1}, \ldots, t_{p}\right)$ of tiles can be chosen in such a way that, for each integer $i$, such that $1 \leq i \leq p$, the intersection of the boundary of tile $t_{i}$ with the boundary of the subfigure of $F$ tiled by $\left\{t_{0}, t_{1}, \ldots, t_{i-1}\right\}$ is connected.

Remark 2.3. Let $T$ and $T^{\prime}$ be tilings of a same polygon $P$. If $f_{T}$ and $f_{T^{\prime}}$ are overtilings such that, for a vertex $v_{0}$ of the boundary of $P, f_{T}\left(v_{0}\right)=f_{T^{\prime}}\left(v_{0}\right)$, then for each vertex $v$ of the boundary of $P, f_{T}(v)=f_{T^{\prime}}(v)$.

The use of the overtilings is a main method to study the tilings. Interesting examples (although the notion of overtiling is not always clearly presented) are developed in [CL], [T], [P], [KK], and [LR]. 
Nevertheless, generally, the use of the overtilings is not easy, since the tiling group is complex. Thus, quotient groups are used, in order to have a group that can be easily described. To obtain such a quotient group, it suffices to exhibit a set $R$ of words such that the contour words of the prototiles are null in the group $G_{R}$. In this case, we have a natural surjection $s$ from $G_{\text {tile }}$ to $G_{R}$.

Let $T$ be a tiling of a polygon $P$, and let $f_{T}$ be an overtiling over $T$. Function $g_{T}$, from the vertices of $F$ to $G_{R}$, defined by $g_{T}=s \circ f_{T}$, is called a tiling projection of tiling $T$.

\section{Tiling with Calissons}

We now study the case when the set of prototiles $S_{\text {cal }}=\left\{\mathrm{cal}_{1}, \mathrm{cal}_{2}, \mathrm{cal}_{3}\right\}$ (the set of the calissons), each calisson being a figure formed from two cells with a common edge, called the central edge of the calisson. A set of contour words of the calissons is $R_{\text {cal }}=\left\{a b a^{-1} b^{-1}, a c^{-1} a^{-1} c, c^{-1} b c b^{-1}\right\}$. The tiling group of the calissons (the calisson group) is denoted by $G_{\text {calisson }}$.

This instance was treated by Thurston [T] using geometrical arguments. We give a purely algebraic presentation of its method, using the notions introduced in the previous section. The main point is the use of a height function to get an order on the tilings, which is specific from the set $S_{\text {cal }}$ of prototiles.

\subsection{Order on the Tilings of a Polygon}

For the calissons, the group $G_{R}$, with $R=\left\{a b^{-1}, a c^{-1}\right\}$, is used. If $a b^{-1}=a c^{-1}=1$, then $a=b=c$, which yields that $a b a^{-1} b^{-1}=a c^{-1} a^{-1} c=c^{-1} b c b^{-1}=1$. Thus the group $G_{R}$ can be seen as a quotient group of the calisson group. Let $P$ be a polygon and fix a vertex $v_{0}$ of the boundary of $P$. For each tiling $T$ of $P, g_{T}$ denotes the only tiling projection of $T$ such that $g_{T}\left(v_{0}\right)=1$.

Notice that for each element $g$ of $G_{R}$, there exists a unique integer $m$ such that $g=a^{m}$. Integer $m$ is called the height of $g$ (denoted by $h(g)$ ). For each tiling $T$ of $P$, the height $h_{T}(v)$ of a vertex $v$ of $V_{T}$ according to $T$ is defined by $h_{T}(v)=h\left(g_{T}(v)\right)$.

The set of the tilings of $P$ can be ordered as follows: $T \leq T^{\prime}$ if, for each vertex $v$ of $P, h_{T}(v) \leq h_{T^{\prime}}(v)$. This ordered set has been studied completely in [STCR].

\subsection{Local Rotations and Key-Lemma}

Let $T$ be a tiling of a polygon $P$ such that three tiles of $T$ form a tiling of a hexagon $H_{x}$ centered in a vertex $x$. A local rotation (see Fig. 2 ) is the substitution of these tiles by the three tiles which form the other tiling of $H_{x}$. A new tiling $T^{\prime}$ is obtained. For each vertex $v$ of $P$, except $x, h_{T}(v)=h_{T^{\prime}}(v)$ and $\left|h_{T}(x)-h_{T^{\prime}}(x)\right|=3$.

Lemma 3.1 (Key-Lemma). Let $T$ be a tiling of a polygon $P$, let $M$ denote the height (according to $T$ ) of a highest vertex of $G_{T}$, let $M^{\prime}$ denote the height of a highest vertex of the boundary of $P$, and let $x$ be a vertex such that $h_{T}(x)=M$. 


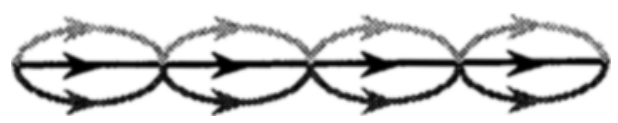

Fig. 2. The Cayley graph of group $G_{R}$.

If $M>M^{\prime}$, then the hexagon $H_{x}$ formed from the six cells having $x$ for common vertex is covered by three tiles of $T$.

Proof. The edge of $C_{\text {triangle }}$ from $x$ to $x a$ (resp., $x b, x c$ ) cannot be an edge of $G_{T}$, since in this case, $h_{T}(x a)$ (resp., $h_{T}(x b), h_{T}(x c)$ ) is equal to $M+1$, which contradicts the maximality of $M$. Thus this edge is the central edge of a tile of $T$.

Corollary 3.2. Let $T$ be a minimal (for the order defined in Lemma 3.1) tiling of a polygon $P$ and let $M$ be the maximal height of a vertex of $P$ according to $T$. There exists $a$ vertex $v$ of the boundary of $P$ such that $h_{T}(v)=M$.

Proof. If it was false, the hypothesis of the key-lemma would hold, and consequently a tiling $T^{\prime}$, which contradicts the minimality of $T$, could be obtained from $T$ using a local rotation.

\subsection{Algorithm of Tiling}

From Corollary 3.2, we deduce an algorithm which constructs a minimal tiling $T$ of the polygon given as input (if such a tiling exists).

\section{Algorithm of Tiling with Calissons}

Input: A polygon $P$ of the triangular lattice formed from $n$ squares.

Initialization: Arbitrarily choose a vertex $v_{0}$ of the boundary of $P$. For each vertex $v$ of the boundary of $P$, compute $g_{T}(v)$ and $h_{T}(v)$, where $g_{T}$ is a tiling projection such that $g_{T}\left(v_{0}\right)=1$ and $h_{T}$ is a height deduced from $g_{T}$.

Main loop: Let $v$ be a highest vertex of the boundary. Place a tile included in $P$ whose central edge (seen as an edge of $G_{\text {triangle }}$ ) is incoming in $v$. Remove the two covered cells from $P$, and update $P$, the boundary of $P$, and functions $g_{T}$ and $h_{T}$.

Repeat these instructions until either $P$ is empty (i.e., a tiling is constructed) or a contradiction appears in the definition of $g_{T}$ (i.e., there is no tiling).

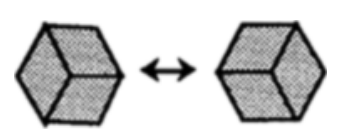

Fig. 3. A rotation of a tiling with calissons. 

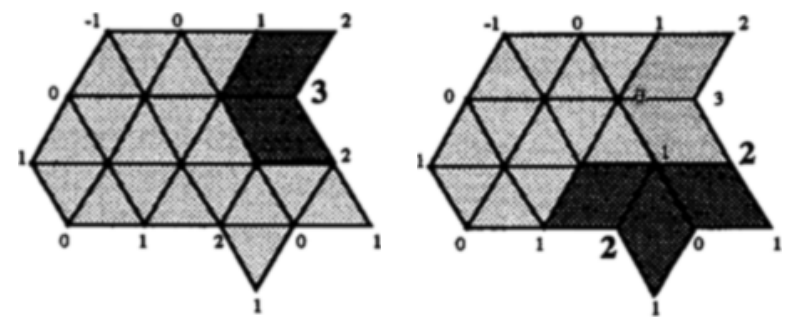

Fig. 4. Algorithm of tiling with calissons.

Since all the updates are local operations, this algorithm can be implemented so as to have a linear running time. We can claim the following theorem:

Theorem 3.3 (W. P. Thurston). A polygon of $\Lambda$ formed from $n$ cells can be tiled by calissons, or proved not to be tileable in time linear in $n$.

\section{Tiling with Leaning Bars and Triangles}

In this section, we extend the method used for the tilings with calissons to another set of prototiles. Fix a positive integer $m$. The set of prototiles that we will use is $\left\{b_{1}, b_{2}, b_{3}, b_{4}, b_{5}, b_{6}, t r_{1}, t r_{2}\right\}$ (see Fig. 3), where:

(a) $b_{1}, b_{2}, b_{3}, b_{4}, b_{5}$, and $b_{6}$ are parallelograms, whose sides have lengths 1 and $m$, these parallelograms being formed from $2 m$ triangular cells. They are called leaning bars.

(b) $t r_{1}$ and $t r_{2}$ are equilateral triangles of side $m$, formed from $m^{2}$ triangular cells.

\subsection{Group $G_{\text {tricolored }}$}

Tilings of polygons generated by those prototiles can be projected on group $G_{\text {tricolored }}=$ $G_{R}$, with $R=\left\{a^{m}, b^{m}, c^{m}\right\}$, since the contour words of the prototiles are null in $G_{\text {tricolored }}=G_{R}$. Cayley Graph $C_{\text {tricolored }}$ is a graph whose elementary cycles have length $m$. Each vertex of $C_{\text {tricolored }}$ is an element of three elementary cycles, the edges of each of these cycles having the same label (see Fig. 6).

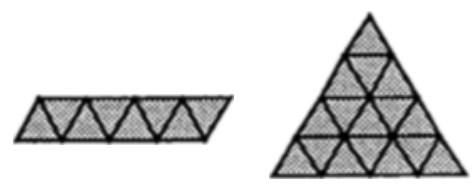

Fig. 5. A leaning bar and a triangle (with $m=4$ ). 


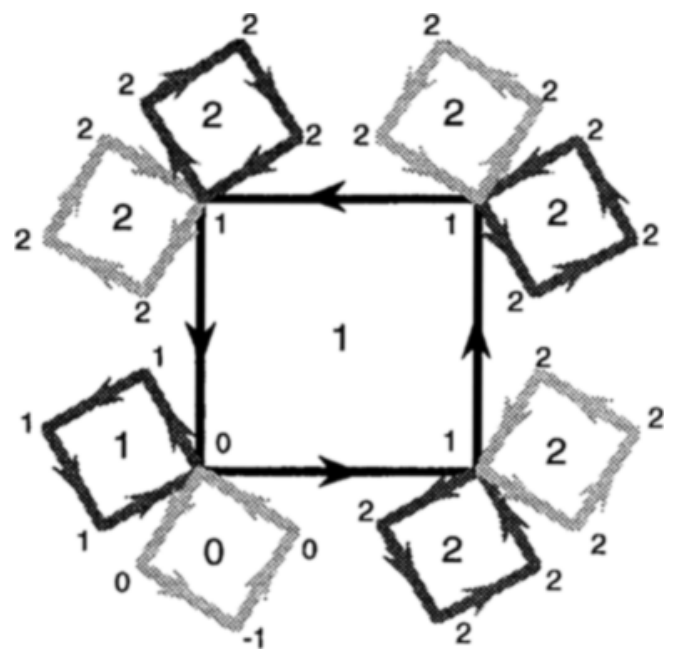

Fig. 6. A part of the Cayley graph of group $G_{\text {tricolored }}$ (with $m=4$ ) with an associated height.

An integer value (which will be called the height) can be associated to each element of $G_{\text {tricolored }}$ as follows: we first attribute a height value to the elementary cycles of $G_{\text {tricolored, }}$, according to the following rules: for each cycle $C$ of height $h$, there exists a unique cycle $C^{\prime}$ of height $h-1$ which share an element $g_{0}$ with $C$, the height of all the other cycles which share $g_{0}$ with $C$ is $h$ and all the cycles which are joined with $C$ by an element $g_{1}$ such that $g_{1} \neq g_{0}$ are cycles of height $h+1$. Thus, for each element $g$ of $G_{\text {tricolored }}$, two of the three elementary cycles containing $g$ have the same height, say $h$, and the height of the third cycle containing $g$ is $h-1$. Value $h-1$ becomes the height of the element $g$.

Such a height function is neither unique nor canonical, but it can easily be constructed step by step, applying the above rules.

Notice that:

(a) the height of all the elements of an elementary cycle of height is $h$ is also $h$, except for one element (the lowest element of the cycle) whose height is $h-1$;

(b) each element of $G_{\text {tricolored }}$ is the lowest element of exactly two cycles which include this element; and

(c) if we take two neighbors of a fixed element $g$ of $G_{\text {tricolored }}$ which are not on the same elementary cycle, then at least one of the neighbors is (strictly) higher than $g$.

As in Section 3, given a tiling $T$ of a polygon $P$ with a fixed vertex $v_{0}$ of the boundary, the height $h_{T}(v)$ of each vertex $v$ of a cell of $P$ is defined saying that $h_{T}(v)$ is the height of $g_{T}(v)=s \circ f_{T}(v)$, where $f_{T}$ denotes the overtiling of $T$ such that $f_{T}\left(v_{0}\right)=1$ and $s$ denotes the canonical surjection from the tiling group to $G_{\text {tricolored }}$. Notice that $h_{T}$ is not defined for the vertices which are elements of the interiors of the triangles of $T$. 


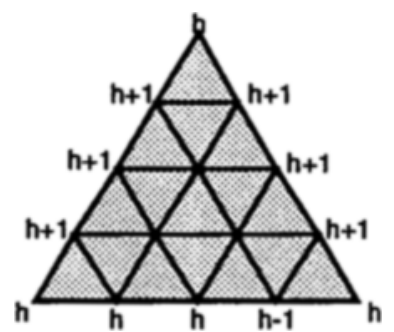

Fig. 7. The heights of the vertices of the boundary of a triangle.

Remark 4.1. The three corners of each triangle $\operatorname{tr}$ of $T$ have the same height, say $h$, the vertices of $t r$ which are on two of the three sides of $t r$ have height $h+1$, and the vertices of the third side have height $h$, except one vertex of height $h-1$ (see Fig. 7).

Let $b$ be a leaning bar of $T$. One of the following alternatives hold (see Fig. 8):

(a) The extremities of the large sides of $b$ have the same height, say $h$, and the other vertices of the boundary of $b$ have height $h+1$.

(b) The extremities of a large side of $b$ have the same height, say $h$, the vertices which are on this side have height $h+1$, the other corners of $b$ have height $h-1$ and the vertices of the other side have height $h$.

(c) The extremities of a large side of $b$ have the same height, say $h$, the vertices which are on this side have height $h+1$, the other corners of $b$ have height $h-1$ and the vertices of the other side have height $h-1$, except one vertex of height $h-2$.

\subsection{Order on the Tilings, Local Rotations, and Key-Lemma}

The set of the tilings of $P$ can be ordered as follows: $T \leq T^{\prime}$ if, for each vertex $v$ of $V_{T} \cap V_{T^{\prime}}, h_{T}(v) \leq h_{T^{\prime}}(v)$.

We have two kinds of local rotations:

(a) Assume that there exists a lozenge $L$, formed from $2 m^{2}$ triangular cells of $P$,

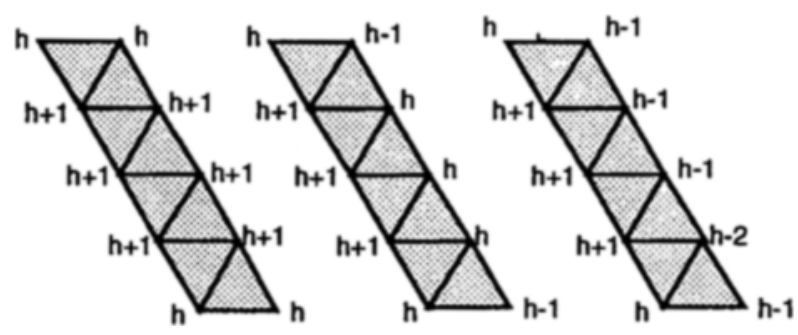

Fig. 8. The possible heights of the vertices of the boundary of a leaning bar. 


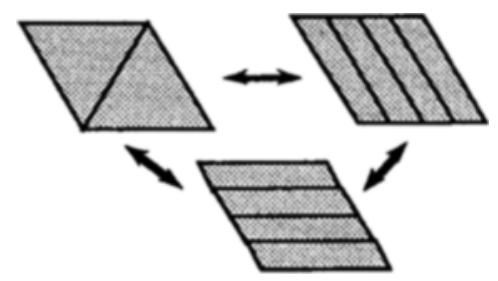

Fig. 9. The lozenge rotations.

such that a subset $L_{T}$ of tiles of $T$ is a tiling of $L$. Notice that lozenge $L$ admits three tilings. Replacing the tiles of $L_{T}$ by other tiles which cover $L$ (see Fig. 9) is our first type of local rotations (the lozenge rotations).

(b) Assume that there exists an isoceles trapeze $R$, formed from $m(m+1)$ triangular cells of $P$, such that a triangle and a leaning bar of $T$ form a tiling of $R$. Replacing these two tiles of $T$ which cover $R$ by the two other tiles which can cover $R$ is our second type of local rotations (the trapeze rotations) (see Fig. 10).

Lemma 4.2 (Key-Lemma). Let $T$ be a tiling of a polygon $P$, let $M$ denote the height of a highest vertex of $V_{T}$, let $M^{\prime}$ denote the height of a highest vertex of the boundary of $P$, and let $x$ be a vertex such that $h_{T}(x)=M$.

If $M>M^{\prime}$, then there exists a lozenge $L_{x}$ with sides of length $m$ (or an isoceles trapeze $R_{x}$ whose little basis has length at most $m-1$ and whose nonparallel sides have length $m$ ) tiled by tiles of $T$, such that $x$ is an interior vertex of $L_{x}\left(\right.$ or $\left.R_{x}\right)$, and the height of all the interior vertices of $L_{x}\left(\right.$ or $\left.R_{x}\right)$ which are in $V_{T}$ is equal to $M$.

Proof. From Remark 4.1, if $x$ is on the boundary of a tile $t i$ of $T$ and $h_{T}(x)=M$, then $x$ cannot be a corner of $t i$. Moreover, the height of the extremities of the side of $t i$ containing $x$ is $M-1$, and all the other vertices of this side have height $M$.

The hypothesis: $M>M^{\prime}$ yields that $x$ is on the boundary of two tiles, say $t i$ and $t i^{\prime}$. These tiles share a complete side since otherwise, the height the corner of one tile would be $M$, and, consequently, this tile would have vertices of height $M+1$.

Thus if at least one of the tiles $t i$ or $t i^{\prime}$ is a triangle, the proof is finished. If tiles $t i$ and $t i^{\prime}$ both are leaning bars, then $t i$ and $t i^{\prime}$ are translated copies of the same prototile, since otherwise a corner of a tile of height $M$ appears.

Let $P_{2}$ be the parallelogram tiled by $t i$ and $t i^{\prime}$. If $m=2$, we state $L_{x}=P_{2}$. For $m>2$, remark that, necessarily, the height of vertices of a large side of $P_{2}$ (except the extremities) is $M$, since at least one of the tiles, $t i$ or $t i^{\prime}$, is on the case (a) of Remark 4.1.

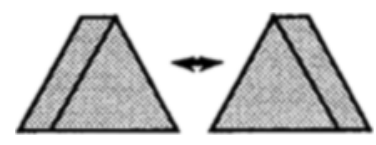

Fig. 10. The trapeze rotations. 
Since $M>M^{\prime}$, those vertices are in the interior of $P$, and consequently are on the large side of another tile $t i^{\prime \prime}$ of $T$. If $t i^{\prime \prime}$ is a triangle, a trapeze $R_{x}$ is obtained which finishes the proof. Otherwise, we can repeat the argument for the parallelogram $P_{3}$ tiled by $t i, t i^{\prime}$, and $t i^{\prime \prime}$, and so on, until either a trapeze is obtained or a parallelogram $P_{m}$ is obtained, and we state $L_{x}=P_{m}$.

Corollary 4.3. Let $T$ be a minimal tiling of a polygon $P$ and let $M$ be the maximal height of a vertex of $P$ according to $T$. There exists a vertex $v$ of the boundary of $P$ such that $h_{T}(v)=M$.

Proof. Assume that $T$ is a minimal tiling of $P$ which does not satisfy this corollary. Let $x$ be a vertex of $P$ such that $h_{T}(x)=M$. We can apply the key-lemma.

If $x$ is an element of the interior of a lozenge $L_{x}$ satisfying this lemma, then a lozenge rotation on $L_{x}$ easily gives a tiling $T^{\prime}$ such that $T^{\prime}$ is lower than $T$, which is a contradiction.

If $x$ is an element of the interior of an isoceles trapeze $R_{x}$ satisfying the lemma, then the height of the vertices of a side of $R_{x}$ of length $m$ is $M$ (except the height of the extremities of this side). Let $x^{\prime}$ denote such a vertex. If $x^{\prime}$ is an element of the interior of a lozenge $L_{x^{\prime}}$ satisfying the lemma, a contradiction is obtained as above. Otherwise, $x^{\prime}$ is an element of the interior of an isoceles trapeze $R_{x^{\prime}}$ satisfying the lemma. Let $P_{x, x^{\prime}}$ be the reunion of $R_{x}$ and $R_{x^{\prime}}$. Polygon $P_{x, x^{\prime}}$ is a parallelogram with small sides of length $m$ and larges sides of length $m^{\prime}$, with $m^{\prime}>m$. We easily verify that the tiling $T^{\prime \prime}$, obtained from $T$ replacing the two triangles and the $m^{\prime}-m$ leaning bars which cover $P_{x, x^{\prime}}$ by the $m$ bars which are translated copies of the same prototiles and form a tiling of $P_{x, x^{\prime}}$, is lower than $T$, which is a contradiction.

Let $T$ be a minimal tiling of minimal height of a polygon $P$ and let $v$ denote a highest vertex of the boundary of $P$. Corollary 4.3 gives at most two possible alternatives (a triangular tile $t r_{0}$ or a leaning bar $b_{0}$ ) for the tile of $T$ a side of which contains vertex $v$. How can the good tile be chosen?

If this tile if $t r_{0}$, then the height of a vertex $v^{\prime}$ of another side of $t r_{0}$ is maximal. If $v^{\prime}$ is on the boundary of $P$, we easily see that $b_{0}$ is not included in $P$, thus tile $t r_{0}$ is forced. Otherwise, vertex $v^{\prime}$ is on the boundary of another tile $t^{\prime}$ of $T$, which no contradiction with Corollary 4.3. The tiles $t r_{0}$ and $t^{\prime}$ cover either a lozenge $L$ (if $t^{\prime}$ is a triangle) or an isoceles trapeze $R$ (if $t^{\prime}$ is a translated copy of $b_{0}$ ). Using a local rotation, a tiling $T^{\prime}$ containing the bar $b_{0}$ is obtained.

That means that when two possible alternatives arise, we can always choose to put the leaning bar. This fact guarantees that the following algorithm constructs a tiling $T$ of minimal height, when the polygon can be tiled.

\section{Algorithm of 'Tiling with Leaning Bars and Triangles}

Input: A polygon $P$ of the triangular lattice formed from $n$ triangular cells.

Initialization: Arbitrarily choose a vertex $v_{0}$ of the boundary of $P$. For each vertex $v$ of the boundary of $P$, compute $g_{T}(v)$ and $h_{T}(v)$, where $g_{T}$ is a tiling projection on $G_{\text {tricolored }}$ such that $g_{T}\left(v_{0}\right)=1$ and $h_{T}$ denotes the height induced by an arbitrarily fixed height of $G_{\text {tricolored }}$ (for instance, the height of Fig. 6). 
Main loop: Let $v$ be a highest vertex of the boundary. If it is possible, place a leaning bar in $P$ with $v$ on its boundary and no contradiction with Corollary 4.3. Otherwise place a triangle in $P$ with $v$ on its boundary and no contradiction with Corollary 4.3.

Remove the covered cells from $P$, and update the boundary of $P$ and functions $g_{T}$ and $h_{T}$.

These instructions are repeated until either $P$ is empty (in this case, a tiling is constructed) or a contradiction or an impossibility appears (in this case, there is no tiling).

Since all the updates are local operations, this algorithm can be implemented so as to have a linear running time. We can claim the following theorem:

Theorem 4.4. A polygon $P$ of the triangular lattice formed from $n$ cells can be tiled with leaning bars and triangles formed from four cells, or proved not to be tileable in time linear in $n$.

\section{Tiling with Leaning Bars}

In this section, the set of prototiles is $\left\{b_{1}, b_{2}, b_{3}, b_{4}, b_{5}, b_{6}\right\}$. The method is a little different from that used for the other sets of prototiles, since the vertices of maximal height of a minimal tiling (using height and order of Section 4) can all be interior. This situation has to be studied carefully.

We need a new notation. Let $x$ and $y$ be distinct elements of $\{a, b, c\}$. We say that a tile $t i$ is a tile of type $x^{m} y$ if $t i$ admits $x^{m} y x^{-m} y^{-1}$ for contour word. Hence, we have six possible types: $a^{m} b, a^{m} c, b^{m} a, b^{m} c, c^{m} a$, and $c^{m} b$.

We need two kinds of rotations: the lozenge rotations of Section 4 which do not use triangular tiles and local rotations of a new type (called the cubic rotations) involving $m+2$ tiles and $2 m(m+2)$ cells. A cubic rotation is presented in Fig. 11 .

Lemma 5.1. Let $T$ be a minimal tiling of a polygon $P$, let $M$ denote the height of a highest vertex of $P$, and let $M^{\prime}$ denote the height of a highest vertex of the boundary of $P$. Assume that $M \neq M^{\prime}$ and let $x$ be a vertex of $P$ such that $h_{T}(x)=M$.

Then there exists a lozenge $L_{x}$ with sides of length $m$, tiled by tiles of $T$, such that $x$ is an interior vertex of $L_{x}$, the height of all the interior vertices of $L_{x}$ is equal to $M$, the height of the comers of $L_{x}$ is equal to $M-2$, and the height of the other vertices of boundary of $L_{x}$ is equal to $M-1$.

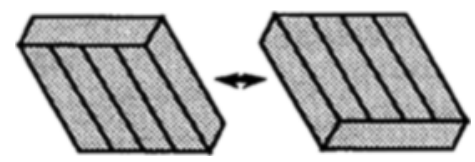

Fig. 11. A cubic rotation. 
Proof. The hypothesis: $M>M^{\prime}$ yields that $x$ is on the boundary of two tiles, say $t i$ and $t i^{\prime}$. These tiles share a complete side since otherwise, the height of the corner of one tile would be $M$, and, consequently, this tile would have vertices of height $M+1$. If tiles $t i$ and $t i^{\prime}$ both are leaning bars, then $t i$ and $t i^{\prime}$ are translated copies of the same prototile, since otherwise a comer of a tile of height $M$ also appears.

Let $P_{2}$ be the parallelogram tiled by $t i$ and $t i^{\prime}$. If $m=2$, we state $L_{x}=P_{2}$. For $m>2$, remark that, necessarily, the height of vertices of a large side of $P_{2}$ (except the extremities) is $M$, since at least one of the tiles, $t i$ or $t i^{\prime}$, is on the case (a) of Remark 4.1.

Since $M>M^{\prime}$, those vertices are in the interior of $P$, and consequently are on the large side of another tile $t i^{\prime \prime}$ of $T$. We can repeat the argument for the parallelogram $P_{3}$ tiled by $t i, t i^{\prime}$, and $t i^{\prime \prime}$, and so on, until a parallelogram $P_{m}$ is obtained, and we state $L_{x}=P_{m}$.

Since $T$ is minimal, the tiling $T^{\prime}$, obtained from $T$ by a lozenge rotation on $L_{x}$, is not lower than $T$, which yields the conditions announced for the heights on the boundary of $L_{x}$.

Lemma 5.2. Let $T$ be a minimal tiling of a polygon $P$, let $M$ denote the height of $a$ highest vertex of $P$, and let $M^{\prime}$ denote the height of a highest vertex of the boundary of $P$.

Assume that $M>M^{\prime}$ and there exists a lozenge $L$, tiled by tiles of $T$, a contour word of which is $a^{m} b^{m} a^{-m} b^{-m}$, such that the height of all the interior vertices of $L$ is equal to $M$.

Then there exists a sequence $\left(L_{1}, L_{2}, \ldots, L_{p}\right)$ (called a zigzag) of translated copies of $L$ such that (see Fig. 12):

(i) there exists an integer $i$ such that $1 \leq i \leq p$ and $L_{i}=L$;

(ii) the left side or the lower side of $L_{1}$ is included in the boundary of $P$;

(iii) the right side or the upper side of $L_{p}$ is included in the boundary of $P$;

(iv) for each integer $i$ such that $1 \leq i<p, L_{i}$ and $L_{i+1}$ share exactly one side, which is either the right side of $L_{i}$ and the left side of $L_{i+1}$ or the upper side of $L_{i}$ and the lower side of $L_{i+1}$; and

(v) for each integer $i$ such that $1 \leq i<p, L_{i}$ is tiled by $m$ tiles of $T$.

Proof. Since $T$ is minimal, the height of the vertices of the sides of $L$ (except the extremities) is $M-1$ and the height of the corners of $L$ is $M-2$, since otherwise a tiling $T^{\prime}$, which is lower than $T$, would be created, using a lozenge rotation.

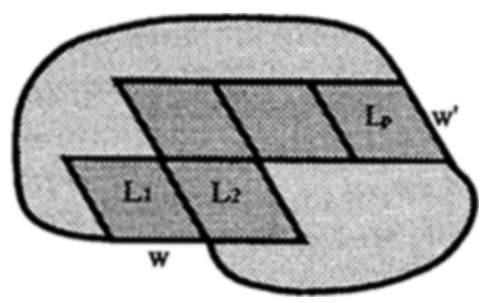

Fig. 12. A zigzag of lozenges. 
If the right side or the upper side of $L$ is included in the boundary of $P$, then state $L=L_{p}$. Otherwise, let $v$ be any vertex of the right side or the upper side of $L$, except the upper right corner of $L$, and let $v^{\prime}$ be one of the two vertices of $P$ which are not in $L$. If the edge whose extremities are $v$ and $v^{\prime}$ is an edge of $G_{T}$ (i.e., this edge crosses no tile of $T$ ), then $h_{T}\left(v^{\prime}\right)=M$, thus there exists a lozenge $L^{\prime}=L_{v^{\prime}}$ satisfying the previous lemma. We easily see that $L^{\prime}$ is necessarily the translated copy of $L$ whose left side is the right side of $L$. Thus, lozenge $L^{\prime}$ can be taken as the successor of $L$ in the zigzag.

Thus, now, we only have to treat the case when each edge as above crosses a tile of $T$. This yields that there exists a tile $t i$ of $T$ whose left side is the right side of $L$, and there exists a tile $t i^{\prime}$ of $T$ whose lower side is the upper side of $L$. If $t i$ is a tile of type $b^{m} a$, then the height of the vertices of the right side of $t i$ (except the extremities) is $M$, thus there exists a lozenge $L^{\prime}$ satisfying the previous lemma, which is necessarily the translated copy of $L$ whose left side is the right side of $L$ and, consequently can be taken as the successor of $L$ in the zigzag. The same argument can be used if $t i^{\prime}$ is a tile of type $a^{m} b$.

Thus, a difficulty arises only when $t i$ is a tile of type $b^{m} c$, and $t i^{\prime}$ is a tile of type $a^{m} c$. But, in such as case, a tiling $T^{\prime}$, which is lower than $T$, would be created, using a cubic rotation replacing tiles $t i, t i^{\prime}$, and the tiles which cover $L$. This contradicts our hypothesis.

This argument can be repeated to construct the successor of $L^{\prime}$, and so on until the right side or the upper side of a lozenge is on the boundary of $P$. Afterwards, the beginning of the zigzag is constructed by the same way.

Corollary 5.3. For each minimal tiling of $P, M \leq M^{\prime}+1$.

Proof. Obvious.

Let $R_{a}=\{b, c\}$. A tiling projection can be defined on $G_{a}=G_{R_{a}}$, since all the contour words of the leaning dominoes are null in $G_{a}$. Group $G_{a}$ is isomorphic to the additive group $\mathbb{Z}$ : since $b=c=1$, for each element $g$ of $G_{a}$, there exists a unique integer $m$ such that $g=a^{m}$ Integer $m$ is called the $a$-height of $g$ (denoted by $h a(g)$ ). The height obtained by the tiling projection of a tiling $T$ on $C_{a}$ is denoted by $h a_{T}$. The $b$-height $h b_{T}$ and the $c$-height $h c_{T}$ are defined by the same way.

Lemma 5.4. Let $T$ be a tiling of a polygon $P$ and let $v$ and $v^{\prime}$ be two vertices of $P$. If there exists an edge of the triangular lattice from $v$ to $v^{\prime}$ whose label is $a$ or $b$, then $h c_{T}(v) \geq h c_{T}\left(v^{\prime}\right)$.

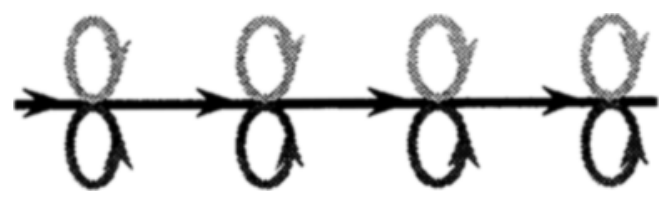

Fig. 13. The Cayley graph of $G_{a}$. 
Proof. Assume that the edge from $v$ to $v^{\prime}$ is labeled by $a$. If this edge crosses no tile of $T$, then $h c_{T}\left(v^{\prime}\right)=h c_{T}(v)$. Otherwise, this edge crosses a tile $t i$ of type $b^{m} a, c^{m} a, b^{m} c$, or $c b$. If $t i$ is of type $b^{m} a$ or $c^{m} a$, then $h c_{T}\left(v^{\prime}\right)=h c_{T}(v)$. If $t i$ is of type $b^{m} c$ or $c^{m} b$, then $h c_{T}\left(v^{\prime}\right)=h c_{T}(v)-1$.

The case when the edge from $v$ to $v^{\prime}$ is labeled by $b$ is treated in the same way.

Lemma 5.5. Let $P$ be a polygon such that the heights $h_{T}$, $h a_{T}, h b_{T}, h c_{T}$ are constructed on the boundary of $P$ with no contradiction. Let $M^{\prime}$ be the maximal height (for $h_{T}$ ) of a vertex of the boundary of $P$, and let $w$ and $w^{\prime}$ be two vertices of the boundary of $P$ such that $h_{T}(w)=h_{T}\left(w^{\prime}\right)=M^{\prime}$ and $h c_{T}(w)=h c_{T}\left(w^{\prime}\right)$.

Assume we can tile, with no contradiction for the heights, a zigzag $\left(L_{1}, L_{2}, \ldots, L_{p}\right)$ such that:

(i) for each integer $i$ such that $1 \leq i \leq p, a^{m} b^{m} a^{-m} b^{-m}$ is a contour word of $L_{i}$;

(ii) for each integer $i$ such that $1 \leq i \leq p$, each interior vertex of $L_{i}$ has height $M^{\prime}+1$

(iii) vertex $w$ is a vertex of either the left side or the lower side of $L_{0}$; and

(iv) vertex $w^{\prime}$ is a vertex of either the right side or the upper side of $L_{p}$.

Then, for each minimal tiling $T$ of $P$, for each integer $i$, with $1 \leq i \leq p, T$ contains $m$ tiles which tile $L_{i}$.

Proof. Obviously, there exists a path $\left(v_{0}, v_{1}, \ldots, v_{q}\right)$ of vertices of $P$ such that:

(i) $v_{0}=w$ and $v_{q}=w$;

(ii) for each integer $i$ such that $0<i<p$, vertex $v_{i}$ is an interior vertex of polygon $L_{1} \cup P_{2} \cup \cdots \cup L_{p}$; and

(iii) for each integer $i$ such that $0 \leq i<p$, the edge from $v_{i}$ to $v_{i+1}$ is labeled by $a$ or $b$.

From Lemma 4.4, we have $h c_{T}\left(v_{i+1}\right) \leq h c_{T}\left(v_{i}\right)$, thus, since $h c_{T}\left(v_{0}\right)=h c_{T}\left(v_{q}\right)$, all the values $h c_{T}\left(v_{i}\right)$, for $0 \leq i \leq q$, are equal. Thus, if the edge from $v_{i}$ to $v_{i+1}$ is labeled by $a$, either this edge crosses not tile or this edge crosses a tile of type $b^{m} a$ or $c^{m} a$.

Now let $k$ be an integer such that $0 \leq k<p$ and assume that each lozenge $L_{i}$, with $i \leq k$, is covered by tiles of $T$. Let $r$ be the largest integer such that $v_{r}$ is element of $L_{i}$. Assume that the edge from $v_{r}$ to $v_{r+1}$ is labeled by $a$ (the case when the edge from $v_{r}$ to $v_{r+1}$ is labeled by $b$ is treated by a symmetric way). Our hypothesis yields that $h_{T}\left(v_{r}\right)=M^{\prime}$ and the edge from $v_{r} \mathrm{t}-v_{r+1}$ cannot cross a tile of type $c^{m} a$.

Thus, $h_{T}\left(v_{r+1}\right)=M^{\prime}+1$, which is the maximal height of a vertex of $P$. This yields, from Lemma 5.1, that $L_{k+1}$ is covered by tiles of $T$. This finishes the proof, by induction on integer $k$.

The above lemma and Corollary 5.3 guarantee the correctness of the following algorithm. 


\section{Algorithm of Tiling with Leaning Bars}

Input: A polygon $P$ of the triangular lattice formed from $n$ triangular cells.

Initialization: Arbitrarily choose a vertex $v_{0}$ of the boundary of $P$. For each vertex $v$ of the boundary of $P$, compute $g_{T}, h_{T}(v), h a_{T}(v), h b_{T}(v), h c_{T}(v)$ step by step. Those functions are initialized giving value 1 to $v_{0}$.

Main loop: Let $w$ be a highest vertex of the boundary of $P$. If the hypothesis of Lemma 5.3 (or a symmetric version of this lemma) can be satisfied, place some tiles which cover the lozenges of the zigzag found.

Otherwise, place the only tile $t i$ such that $w$ is on a large side of $t i$ and $w$ is a highest vertex of $t i$.

Remove the tiled cells from $P$, and update the boundary of $P$ and functions $g_{T}, h_{T}$, $h a_{T}, h b_{T}, h c_{T}$.

These instructions are repeated until either $P$ is empty (in this case, a tiling is constructed) or a contradiction appears in the definition of $g_{T}$ (in this case, there is no tiling).

The search of a zigzag costs at most $O(n)$ time units since it is very similar to the search of a special leave of a binary tree with at most $n$ vertices (since a lozenge has at most two potential successors for a zigzag, and the number of potential lozenges to explore is lower than $n$ ). Thus this algorithm can be implemented so as to have a quadratic running time. We can claim the following theorem:

Theorem 5.6. A polygon $P$ of the triangular lattice formed from $n$ cells can be tiled with leaning bars, or proved not to be tileable in time quadratic in $n$.

\section{Conclusion}

This paper gives a partial unification of the methods used to get an algorithm of tiling. Thurston [T] and C. and R. Kenyon [KK] have obtained similar results, which can be explained using height functions on Cayley graphs on quotient groups, with "dominoes" and "bars" of the square lattice. Chaboud [C], using a very neighboring height function, gave an algorithm of tiling with "dominoes" on lattices which cannot be seen as supports of Cayley graphs. Underlying algebraic structures do not seem to explain its result.

Is there a better unification? Why can our method not be applied in the hexagonal lattice, where only very partial results have been obtained (see [T], [KR], and [LR])? For which sets of prototiles is there a height function which permits us to give an algorithm of tiling? How can the quotient group be chosen?

All these questions have not received a clear answer yet.

\section{References}

[C] T. Chaboud, Pavages et graphes de Cayley, Ph.D. Thesis, Ecole Normale Supérieure de Lyon, 1995.

[CL] J. H. Conway and J. C. Lagarias, Tiling with polyominoes and combinatorial group theory, J. Combin. Theory Ser. A, 53 (1990), 183-208. 
[KK] C. Kenyon and R. Kenyon, Tiling a polygon with rectangles, Proceedings of 33rd FOCS, 1992 , pp. 610-619.

[KR] C. Kenyon and E. Rémila, Perfect matchings in the triangular lattice, Discrete Math. To appear.

[LR] J. C. Lagarias and D. S. Romano, A polyomino tiling of Thurston and its configurational entropy, J. Combin. Theory Ser. A, 63 (1993), 338-358.

[P] J. Propp, A pedestrian approach to a method of Conway, or a tale of two cities, Internal report, Massachusetts Institute of Technology, 1993.

[R] E. Rémila, Tiling a figure using a height in a tree, Proceedings of the 7th Annual ACM-SIAM Symposium On Discrete Algorithms (SODA) (SIAM, eds.), Philadelphia, PA, 1996, pp. 168-174.

[STCR] N. C. Saldanha, C. Tomei, M. A. Casarin, Jr., and D. Romualdo, Space of domino tilings, Discrete Comput. Geom., 14 (1995), 207-233.

[T] W. P. Thurston, Conway's tiling group, Amer. Math. Monthly (1990), 757-773.

Received July 3, 1996, and in revised form May 14, 1997. 